\title{
Measures of Effect in Epidemiological Research
}

\author{
Giovanni Tripepi $^{\mathrm{a}}$ Kitty J. Jager ${ }^{\mathrm{b}}$ Friedo W. Dekker ${ }^{\mathrm{b}, \mathrm{c}}$ Carmine Zoccali ${ }^{\mathrm{a}}$ \\ ${ }^{a}$ CNR-IBIM, Clinical Epidemiology and Physiopathology of Renal Diseases and Hypertension, Reggio Calabria, Italy; \\ ${ }^{b}$ ERA-EDTA Registry, Department of Medical Informatics, Academic Medical Center, University of Amsterdam, \\ Amsterdam, and ${ }^{\mathrm{C}}$ Department of Clinical Epidemiology, Leiden University Medical Centre, Leiden, The Netherlands
}

\section{Key Words}

Attributable rate $\cdot$ Attributable risk $\cdot$ Incidence rate ratio .

Odds ratio $\cdot$ Relative risk $\cdot$ Risk ratio

\begin{abstract}
The study of the relationship between risk factors and outcomes is important both in etiological and prognostic research. To assess the strength of a given risk factor-outcome relationship we use measures that are calculated in relative and absolute terms. Risk ratio, incidence rate ratio and odds ratio are relative measures of this relationship. Risk difference (or attributable risk) and rate difference (or attributable rate) are absolute measures of the same relationship. Risk difference and rate difference are calculated by subtracting the risk and the incidence rate in exposed individuals from that in unexposed individuals, respectively. The choice of these measures depends on the study aim. Relative measures are commonly used in etiological studies while absolute measures are mainly used in public health research.
\end{abstract}

Copyright $\odot 2010$ S. Karger AG, Basel

\section{Introduction}

Measuring the strength of observed associations between a given risk factor (e.g. blood pressure) and a given outcome (e.g. stroke) is an important goal in epidemiological and clinical research. When we assess these links we take a measure of disease frequency in a group as a basis for the calculation [1]. Thus, we express the strength of the association in relative terms [i.e. as risk ratio, incidence rate ratio or odds ratio (OR)] or in absolute terms [i.e. as risk difference (attributable risk) and rate difference (attributable rate)].

\section{Relative Measures}

\section{Relative Risk}

The relative risk can be calculated as the ratio between two risks (risk ratio, see example 1) or two incidence rates (incidence rate ratio, see example 2).

\section{Example 1}

In a cohort study in end-stage renal disease patients [2] the association of high levels of norepinephrine (NE) with the 3 years' risk of fatal and nonfatal cardiovascular (CV) events was investigated by calculating the ratio be-

Dr. Giovanni Tripepi, CNR-IBIM, Istituto di Biomedicina, Epidemiologia Clinica e Fisiopatologia delle Malattie Renali e dell'Ipertensione Arteriosa c/o Euroline di Ascrizzi Vincenzo

Via Vallone Petrara n. 55/57, IT-89124 Reggio Calabria (Italy)

Tel. +390965 397 010, Fax +390965 26879, E-Mail gtripepi@ibim.cnr.it 
tween the risk of $\mathrm{CV}$ events in patients with $\mathrm{NE}>75$ th percentile (that is, NE $>5.6 \mathrm{nmol} / \mathrm{l}$ ) and in those with $\mathrm{NE}$ below this threshold.

\begin{tabular}{llrr}
\hline & $\begin{array}{l}\text { Patients with } \\
\text { CV events }\end{array}$ & $\begin{array}{l}\text { CV event- } \\
\text { free patients }\end{array}$ \\
\hline NE $\leq 75$ th percentile $(\mathrm{n}=171)$ & 54 & 117 & 171 \\
NE $>75$ th percentile $(\mathrm{n}=57)$ & 29 & 28 & 57 \\
\hline
\end{tabular}

3 years' risk of CV events in the low NE group ( $\leqslant 75$ th percentile): $54 / 171=0.316(31.6 \%)$.

3 years' risk of CV events in the high NE group

(>75th percentile): $29 / 57=0.509(50.9 \%)$.

The risk ratio is: $0.509 / 0.316=1.61$.

The null (neutral) value for the risk ratio indicating that there is no excess or reduced risk is 1 . A risk ratio $<1.0$ means that the risk of the disease is lower in exposed individuals than in unexposed individuals; vice versa a risk ratio $>1.0$ implies that the risk of disease is higher in exposed individuals. Therefore, 1.61 indicates that the risk of CV events is $61 \%$ higher in patients with increased NE levels ( $>75$ th percentile) than in those with relatively low NE levels ( $\leq 75$ th percentile). Together with the point estimate of the risk ratio (1.61), it is also important to provide the confidence interval (CI) which is a measure of the precision of the same estimate. In the example above, the $\mathrm{CI}$ for the risk ratio of $\mathrm{CV}$ events in patients with NE $>75$ th percentile as compared to those with NE $\leq 75$ th percentile was $1.15-2.26$. This interval represents the range of values in which the true risk ratio (that is, the risk ratio in the theoretical population of all dialysis patients worldwide) is likely to lie with a probability of $95 \%$. To be statistically significant $(\mathrm{p}<0.05)$, a risk ratio should have a $95 \%$ CI not including 1.0. Therefore, the risk ratio of 1.61 (95\% CI: 1.15-2.26) is statistically significant.

\section{Example 2}

Fellström et al. [3] investigated the effect of rosuvastatin on the risk of CV events in patients undergoing hemodialysis. They reported the relative risk of CV outcomes in patients treated with rosuvastatin versus those untreated in terms of incidence rate ratio.

Incidence rate of $\mathrm{CV}$ outcomes events/1,000 person-years at risk

On treatment with rosuvastatin

On treatment with placebo

95
The incidence rate ratio is the incidence rate of the event of interest in the exposed group (rosuvastatin group) divided by the incidence rate of the same event in the unexposed group (placebo group). In the study of Fellström et al. [3], the incidence rate ratio was calculated as: $92 / 95=0.96$ (95\% CI: 0.84-1.11). Thus, in this study the incidence rate of $\mathrm{CV}$ events is $4 \%$ lower in patients on rosuvastatin than in those on placebo but this small risk reduction largely fails to achieve the statistical significance (the 95\% CI included 1.0).

\section{OR (See Example 3)}

The odds are typically used by sports gamblers. For example, we may read that the odds for England winning the World Cup are 30 to 1 against. Here odds means that it is expected that the English team is 30 times more likely to lose than to win the World Cup competition. In a case-control study the OR is calculated as the ratio of the number of exposed individuals divided by the number of unexposed individuals in cases and controls, respectively. An important property of the OR is that the OR of the exposure equals the OR of the disease and vice versa [4]. Therefore, an OR $<1.0$ implies that the odds of the disease are lower in exposed individuals than in unexposed individuals; vice versa an OR $>1.0$ implies that the odds of the disease are higher in exposed individuals.

\section{Example 3}

Matteucci et al. [5] designed a case-control study for comparing echocardiographic alterations in children with chronic renal failure and in a group of age- and sexmatched healthy control children. In the study they included 156 children with chronic renal failure (cases) and 133 healthy children (controls). Among the 156 cases, 33 had echocardiographic evidence of eccentric left ventricular hypertrophy (LVH) and 123 did not, while among the 133 controls 3 had eccentric LVH and 130 did not.

Odds of eccentric LVH in children with chronic renal

failure: $33 / 123=0.27$.

Odds of eccentric LVH in children without chronic renal

failure: $3 / 130=0.023$.

The OR is: $0.27 / 0.023=11.6$.

An odds ratio of 11.6 means that the odds of eccentric $\mathrm{LVH}$ are about 12 times higher in children with CRF than in controls or alternatively it means that the OR of chronic renal failure is about 12 times in children with eccentric LVH than in those without such an alteration. This OR was statistically significant because the $95 \%$ CI of the estimate (95\% CI: 3.5-38.9) did not include 1. 


\section{Absolute Measures of Effect}

\section{Risk Difference}

The effect associated with a given treatment can also be expressed in terms of absolute risk difference. The calculation is the difference between the risk of a given event in the control group and the risk of the same outcome in the treated group or vice versa. In a recent study, Dixon et al. [6] investigated the effect of dipyridamole plus aspirin on hemodialysis graft patency. The incidence of primary unassisted patency at 1 year was $28 \%$ in the dipyridamole-aspirin group and $23 \%$ in the placebo group. The risk difference for the primary end point (i.e. patency without thrombosis or requirement for intervention) between patients in the active arm of the study and those on placebo was $0.28-0.23=0.05$ ( $95 \%$ CI: -0.01 to 0.11 ), i.e. the absolute risk difference attributable to the combined treatment dipyridamole plus aspirin was rather low (only 5\%) and not significant (the 95\% CI included 0\%). By using the risk difference it is possible to calculate the 'number needed to treat' (NNT) to prevent 1 adverse event. NNT is considered to be a clinically very useful measure. In the study of Dixon et al. [6], the number of patients to be treated to prevent one fistula thrombosis episode in 1 year is calculated as the inverse of the absolute risk difference: $1 / 0.05$ which is equal to 20 . In other words, we ought to treat 20 patients with dipyridamole plus aspirin to prevent 1 thrombotic episode/year. However, given the fact that in the study of Dixon et al. [6] the risk difference was not statistically significant, the NNT calculation is of limited value in this particular context because the study failed to document a superiority of the drug regimen over placebo.

\section{Rate Difference}

To describe the calculation of the rate difference we consider a hypothetical example investigating the occurrence of cancer in a cohort of 15,326 men employed by a chemistry company (example 4).

\section{Example 4}

\begin{tabular}{lllrc}
\hline $\begin{array}{l}\text { Duration of } \\
\text { employment }\end{array}$ & $\begin{array}{l}\text { Number } \\
\text { of cases }\end{array}$ & $\begin{array}{l}\text { Person- } \\
\text { years }\end{array}$ & Rate & \multicolumn{1}{l}{$\begin{array}{l}\text { Rate difference } \\
(95 \% \mathrm{CI})\end{array}$} \\
\hline$<1$ year & 44 & 40,056 & 11 & 0 \\
$1.0-1.9$ years & 67 & 21,165 & 32 & $21(12-99)$ \\
$2.0-4.9$ years & 19 & 3,105 & 61 & $50(23-78)$ \\
$5.0-9.9$ years & 48 & 5,067 & 95 & $84(57-111)$ \\
$\geq 10$ years & 43 & 4,192 & 103 & $92(61-122)$ \\
\hline
\end{tabular}

In this study, the rate is expressed as the number of cases of cancer per 10,000 person-years. Since individuals with a duration of employment of less than 1 year were those with the lowest rate of cancer, the authors considered these individuals as a reference group. The rate differences (or attributable rate) were calculated as the difference between the incidence rate of cancer in individuals in each category and in those in the reference category. This calculation showed that there was a graded increase in the rate of cancer from the reference category onwards, indicating that the duration of exposure to chemical agents is strongly associated with the rate of cancer. Of note, the excess rate in each category was statistically significant because the $95 \%$ CI do not include 0 .

\section{Conclusions}

Risk ratio (i.e. the ratio between two risks), incidence rate ratio (the ratio between two incidence rates) and $\mathrm{OR}$ (the ratio between two odds) represent relative measures of association between exposure and outcome. The risk difference and the rate difference are absolute measures of the same relationship. The risk difference is frequently used in clinical trials to calculate the NNT, i.e. the number of individuals that we need to treat to prevent 1 adverse event in a given time period.

\section{References}

1 Noordzij M, Dekker FW, Zoccali C, Jager KJ: Measures of disease frequency: prevalence and incidence. Nephron Clin Pract 2010; 115:c17-c20

2 Zoccali C, Mallamaci F, Parlongo S, et al: Plasma norepinephrine predicts survival and incident cardiovascular events in patients with end-stage renal disease. Circulation 2002;105:1354-1359.

3 Fellström B, Jardine A, Schmieder RE, et al: Rosuvastatin and cardiovascular events in patients undergoing hemodialysis. N Engl J Med 2009;360:1395-1407.
4 Selvin S: Statistical Analysis of Epidemiologic Data. New York, Oxford University Press, 1991, p 452.

5 Matteucci MC, Wuhl E, Picca S, et al: Left ventricular geometry in children with mild to moderate chronic renal insufficiency. J Am Soc Nephrol 2006;17:218-226.

6 Dixon BS, Beck GJ, Vazquez MA, et al: Effect of dipyridamole plus aspirin on hemodialysis graft patency. N Engl J Med 2009;360: 2191-2201. 